Research Article

\title{
Stereotactic Body Radiotherapy as a Salvage Therapy after Incomplete Radiofrequency Ablation for Hepatocellular Carcinoma: A Retrospective Cohort Study
}

\author{
Yizhen Fu $\mathbb{D}^{1,2}$ Mian Xi, ${ }^{1,3}$ Yangxun Pan, ${ }^{1,2}$ Jinbin Chen,, Juncheng Wang, ${ }^{1,2}$ \\ Shiliang Liu, ${ }^{1,3}$ Li Xu, ${ }^{1,2}$ Zhongguo Zhou $\mathbb{D}^{1,2}$ Mengzhong Liu, ${ }^{1,3}$ Minshan Chen, ${ }^{1,2}$ \\ Lei Zhao, ${ }^{1,3}$ and Yaojun Zhang ${ }^{1,2}$ \\ ${ }^{1}$ Sun Yat-sen University Cancer Center, State Key Laboratory of Oncology in South China, \\ Collaborative Innovation Center for Cancer Medicine, Guangzhou, Guangdong 510060, China \\ ${ }^{2}$ Department of Liver Surgery, Sun Yat-sen University Cancer Center, Guangzhou, Guangdong 510060, China \\ ${ }^{3}$ Department of Radiation Oncology, Sun Yat-sen University Cancer Center, Guangzhou, Guangdong 510060, China
}

Correspondence should be addressed to Yaojun Zhang; zhangyuj@sysucc.org.cn

Received 3 March 2020; Accepted 12 May 2020; Published 28 May 2020

Academic Editor: Dongfeng Qu

Copyright (c) 2020 Yizhen Fu et al. This is an open access article distributed under the Creative Commons Attribution License, which permits unrestricted use, distribution, and reproduction in any medium, provided the original work is properly cited.

Residual tumor tissue after radiofrequency ablation (RFA) is inevitable in clinical practice, and the optimal management of residual tumor after RFA has not been established. To evaluate the efficiency and toxicity of stereotactic body radiotherapy (SBRT) as a salvage therapy after incomplete RFA for hepatocellular carcinoma (HCC), we retrospectively included 32 HCC patients with an initial incomplete response (iIR) to RFA from May 2011 to August 2018. An iIR was defined as the presence of residual enhancement on CT or MRI one month after RFA treatment. The primary endpoint was local tumor control (LTC); the secondary endpoints included progression-free survival (PFS), overall survival (OS), and toxicity. All patients fulfilled 6 fractions of SBRT as planned, with dosages ranging from $30 \mathrm{~Gy}$ to $54 \mathrm{~Gy}$. The objective response rate (ORR) was 50.0\%. The 1- and 2-year LTC rates were $86.6 \%$ (95\% CI, $74.3 \%$ to $98.9 \%$ ) and $74.7 \%$ (95\% CI, $55.9 \%$ to $93.5 \%$ ), respectively. Fewer times of prior treatments was associated with better LTC (HR $=11.7, P=0.026)$. The 1 - and 2 -year PFS rate were $69.9 \%(95 \% \mathrm{CI}, 53.4 \%$ to $86.4 \%)$ and $52.7 \%$ (95\% CI, 33.1\% to 72.3\%), respectively. A higher Child-Pugh score was the only independent risk factor for tumor progression $(\mathrm{HR}=5.17, P=0.012)$. The 1 - and 3 -year OS rate were $85.6 \%$ and $67.1 \%$, respectively. Only two patients suffered grade 3 adverse events, and none experienced grade 4 or 5 events. In conclusion, for HCC patients confirmed to have an iIR to prior RFA, with compensated liver function, SBRT provided favorable LTC and OS along with acceptable toxicity.

\section{Introduction}

Liver cancer is the sixth most commonly diagnosed cancer and the fourth leading cause of cancer death worldwide in 2018, with approximately 841,000 new cases and 782,000 deaths annually. Hepatocellular carcinoma (HCC) is the most common primary liver cancer, accounting for $75 \%-$ $85 \%$ of cases [1]. For early-stage HCC, transplantation, surgical resection, and ablation are considered as curative treatments [2].
Radiofrequency ablation (RFA) provides comparable results to liver resection with fewer complications in earlystage HCC. Technical success, also known as an initial complete response (iCR), which refers to the thorough coagulation necrosis of the treated lesion, is reported to be associated with tumor control and patient survival $[3,4]$. However, the iCR rate fails to reach $100 \%$ even in case of small HCC lesions, since some HCC lesions are located beneath the liver capsule or adjacent to vessels (leading to the so-called "heat-sink effect") [5]. Residual tumor tissue 
after RFA treatment, or an initial incomplete response (iIR), is inevitable in clinical practice even with skilfully applied ablation techniques and advanced approaches. Several studies have reported an initial failure rate of RFA ranging from $5.2 \%$ to $16.9 \%[3,6-8]$. Most of these patients underwent repeated RFA after the confirmation of an iIR [3-9], yet the underlying causes of RFA failure have not been settled, and the iIR rate of repeated RFA is still approximately $7 \%(6.6$ to $7.8 \%)[7,8]$. Given that an iCR is a major predictor of survival, treatment of the remaining lesion is as essential as treatment of the primary $[3,4]$. Little research has focused on treatment after prior RFA failure, and the optimal management for residual tumor tissue owing to an iIR to RFA has not been established.

Stereotactic body radiation therapy (SBRT) is a treatment modality that involves the delivery of very high individual doses of radiation to tumors with high precision within a single or a small number of fractions [10]. Due to its high geometric precision and accuracy and the consequently lower radiation exposure of nontargeted tissue, along with fair local control and feasible toxicities [11-13], SBRT has emerged as an alternative treatment to conventional therapies. Retrospective studies have shown equivalent overall survival (OS) and superior local control with SBRT compared to RFA $[14,15]$. Since SBRT has been proven to be efficient in small HCC for curative intension, we rationally assume that SBRT should provide fair local control and favor OS if it is applied as a salvage operation to residual lesions after RFA. As far as we know, the outcomes of SBRT in treating residual lesions remain unknown. Thus, in this observational study, we reviewed patients who underwent SBRT after confirmation of an iIR to prior RFA treatment, evaluated the efficiency and toxicity of SBRT, and assessed factors potentially influencing tumor control and patient survival.

\section{Materials and Methods}

2.1. Data Source. This study was approved by the ethics committee of the Sun Yat-sen University Cancer Center (SYSUCC). Informed consent was impossible to obtain because this was a retrospective study. However, written informed consent for the use of data for research purposes was signed before each treatment. We reviewed the medical records of HCC patients who suffered initial incomplete necrosis after RFA and consequently underwent SBRT between May 2011 and August 2018 at the SYSUCC. The patients' baseline information, including age, sex, BMI, and preceding liver-directed treatments, was obtained from clinical records. Imaging data and laboratory investigation results were collected from the database of the SYSUCC.

2.2. Study Population. The object of this study was HCC patients. The diagnosis of HCC was confirmed by biopsy or imaging analysis showing intense contrast uptake during the arterial phase followed by contrast washout in the venous or delayed phase on dynamic computed tomography (CT) or magnetic resonance imaging (MRI).
According to the RFA protocol of the SYSUCC, an early follow-up examination was conducted one month after the RFA operation; thus, an iCR, or technical success of RFA, was defined when the ablated tumor was completely replaced by a necrosis zone with no enhancing tissue at the tumor site, while an iIR, or failed RFA, was defined by the presence of residual enhancement on CT or MRI at the early follow-up time [7,9]. Once an iIR to previous RFA was confirmed, further treatment was proposed by a multidisciplinary team. Tumor characteristics, liver function, patient performance, and comorbidities were considered to develop the best treatment plan. Generally, the multidisciplinary team proposed SBRT to patients with an iIR who experienced repeated tumor recurrence or whose tumor abutted intrahepatic vessels or the liver capsule. Those who were verified as having an IIR to RFA and subsequently underwent SBRT treatment were enrolled in this retrospective study.

The inclusion criteria were as follows: $\geq 18$ years old; Child-Pugh (CP) A liver function; and Karnofsky performance score $\geq 60$ before SBRT implementation. The exclusion criteria were as follows: bilirubin $\geq 3$ times the upper limit of normal; AST or ALT $\geq 6$ times the upper limit of normal; serum creatinine greater than $200 \mu \mathrm{mol} / \mathrm{L}$; international normalized ratio $\geq 1.3$; hemoglobin less than $90 \mathrm{~g} / \mathrm{L}$; platelets less than $80,000 / \mu \mathrm{L}$; clinical ascites; and previous irradiation to the right upper abdomen. Extrahepatic metastasis was permitted.

To investigate whether the tumor location influenced the efficacy of SBRT, we classified the residual HCC nodules based on their intrahepatic locations shown on CT or MRI before the initiation of SBRT. According to their relation to intrahepatic vessels, the target lesions were classified as perivessel HCC lesions and non-perivessel HCC lesions. Perivessel HCC was defined as an index tumor with any contact with first- or second-degree branches of an intrahepatic vessel that was $3 \mathrm{~mm}$ or greater in axial diameter. Subcapsular HCC was defined as a target tumor adjoining the liver capsule on axial or coronal sections with a distance from the hepatic capsule to the tumor margin less than $1 \mathrm{~mm}$. Otherwise, the lesion was considered non-subcapsular [16].

2.3. SBRT Treatment. Patients underwent CT simulation with vacuum pillows used to individually immobilize the torso during radiotherapy. Four-dimensional CT combined with a respiratory gating system was used to enable accurate motion management. The gross target volume (GTV) was exactly coincident with the tumor images or enhancing vessel thromboses on CT/MRI. The clinical target volume (CTV) included a $5 \mathrm{~mm}$ expansion around the GTV. Statistics obtained during free breathing, deep inspiration, and deep expiration were applied to generate an internal target volume (ITV) that accounted for respiratory motion. Finally, to account for mechanical error and unpredictable changes during each fraction, another margin was added to the ITV to form a patient-specific planning target volume (PTV). The maximal allowable dose to $0.5 \mathrm{~mL}$ of the 
esophagus, duodenum, stomach, and bowel was $30 \mathrm{~Gy}$, that to the spinal cord was $27 \mathrm{~Gy}$, and that to the heart was $52.5 \mathrm{~Gy}$. The chest wall received no more than $35 \mathrm{~Gy}$ per $30 \mathrm{~mL}[13,14,17,18]$. Dosimetry was prescribed to the isodose surface covering $99.5 \%$ of the PTV, and regional underdosing was allowed to meet normal tissue limits. Patients received 30-54 Gy radiation in 6 fractions every other day with a 6 to $8 \mathrm{MV}$ X-ray beam applied using the Elekta Precise Treatment System (Elekta AB, Stockholm, Sweden) or the Elekta Versa HD System (Elekta AB, Stockholm, Sweden). Cone-beam CT was performed before each treatment for the patients, and image-guided radiation therapy was used for repositioning.

2.4. Evaluation. Patients underwent triphasic liver MRI or CT scan one month after finishing all SBRT fractions and were followed up every 3 months in the first year and every 6 months thereafter. Along with imaging data, clinical symptoms and signs were evaluated. Blood specimens were collected at each follow-up time to test for blood system disorders, hepatobiliary system disorders, and tumor markers. Patients who showed suspicious clinical or tomography features of extrahepatic metastasis underwent appropriate further imaging examinations for confirmation. When recurrence after SBRT was detected during the followup time, the patient received further local or systemic therapy.

The tumor response after SBRT was assessed according to modified Response Evaluation and Criteria in Solid Tumors (mRECIST) $[2,19]$. Local tumor control (LTC) was defined as the absence of progressive disease within or at the PTV margin, while the presence of progressive disease was considered local tumor progression (LTP). New hepatic lesions that emerged outside the PTV margin were classified as intrahepatic distant recurrence (IDR) [11, 13, 14, 17, 20]. The primary endpoint of this study was LTC, and the secondary endpoints included progression-free survival (PFS), overall survival (OS), and toxicity. PFS was defined as the time from the start of SBRT to the earliest event (i.e., LTP, IDR, or extrahepatic recurrence (ER)) pinpointed on radiology. Adverse events were assessed in the first follow-up procedure by the Common Terminology Criteria for Adverse Events (CTCAE), version 5.0. Dose-limiting toxicity (DLT) was considered any grade 4 or 5 liver, intestinal, or hematopoietic system toxic effects or radiation-induced liver disease (RILD), which consisted of hepatomegaly, anicteric ascites, and elevated alkaline phosphatase $[13,17,21]$.

2.5. Statistical Methods. All recruited patients were enrolled in the calculation of LTC, PFS, and OS. The LTC, PFS, and OS curves were summarized by the Kaplan-Meier method. Raw data for continuous variables, consisting of age, BMI, number of prior treatments, and alpha-fetoprotein (AFP) level and dose to the PTV, were recorded and further converted into categorical data by dichotomizing them by the median values (AFP level: $200 \mathrm{ng} / \mathrm{mL}$ ). Survival curves of different groups were compared by the log rank test for univariate analysis. The effects of covariates on LTC, PFS, and OS were evaluated by the hazard ratio (HR) using Cox proportional hazards regression models. Variables with $P$ values less than 0.1 and those that may have an impact on tumor progression or survival based on clinical experience were included in the multivariate analysis. Continuous variables were compared using the Mann-Whitney test, and categorical variables were compared by the $\chi 2$ test or Fisher's exact test. Statistical significance was evaluated at $P \leq 0.05$, and all analyses were performed using SPSS (version 25.0: SPSS, Inc., Chicago, United States) or R (version 3.5.1: R Foundation, Vienna, Austria).

\section{Results}

3.1. Patients and Treatments. Between May $1^{\text {st }}, 2011$, and August $1^{\text {st }}, 2018$, a total of 58 patients received RFA followed by liver SBRT at the SYSUCC. Among them, 23 were confirmed to have tumor recurrence rather than an iIR to RFA and were therefore excluded. In the remaining 35 patients, 2 had liver metastases, and 1 had intrahepatic cholangiocarcinoma. Finally, 32 patients were included in the study in accordance with the inclusion criteria (Figure 1). The longest follow-up was 5 years. As of April $1^{\text {st }}, 2019$, seven patients had died due to tumor progression, while the rest were treated as censored. The median follow-up time was 24.0 months (1.7 months to 60.0 months).

Hepatitis B virus (HBV) infection was present in 22 $(68.8 \%)$ patients in this study, practically all of whom had received anti-HBV therapy. Treatments for hepatitis $\mathrm{C}$ virus (HCV) were not conducted in the $2 \mathrm{HCV}$-infected patients before SBRT and were executed after completion of the SBRT schedules. Although nearly half of the patients (46.9\%) developed cirrhosis, their liver function remained compensated, and none of them scored 7 or worse on the CP scale. The median tumor diameter was $28 \mathrm{~mm}$. Twenty-three lesions were classified as perivessel HCC, 8 were classified as subcapsular HCC, and 3 were both perivessel and subcapsular HCC. The baseline characteristics of the enrolled patients and the target lesions are listed in Table 1.

3.2. Tumor Control and Overall Survival. An objective response was observed in $8(25.0 \%)$ of 32 lesions one month after completing SBRT treatment. At this time, the best overall responses were observed in $7(21.7 \%)$ patients with complete response (CR) and 1 (3.1\%) patient with partial response (PR). Twenty-one (65.9\%) lesions were assessed as stable disease (SD), and $3(9.3 \%)$ lesions were assessed as progressive disease (PD). During long-term follow-up, LTP was observed in 6 patients, 13 patients reached a sustained $\mathrm{CR}$, and 3 patients reached a sustained $\mathrm{PR}$; the long-term objective response rate (ORR) was $50.0 \%$. The one- and twoyear LTC rates were $86.6 \%$ (95\% confidence interval $(95 \%$ CI), $74.3 \%$ to $98.9 \%$ ) and $74.7 \%$ (95\% CI, $55.9 \%$ to $93.5 \%$ ), respectively (Figure 2(a)). The median time to LTP was not reached. Univariate and multivariate analyses revealed that the number of prior treatments was associated with LTC: those who had received more than three prior treatments were more likely to suffer LTP $(H R=11.7, P=0.026$, 


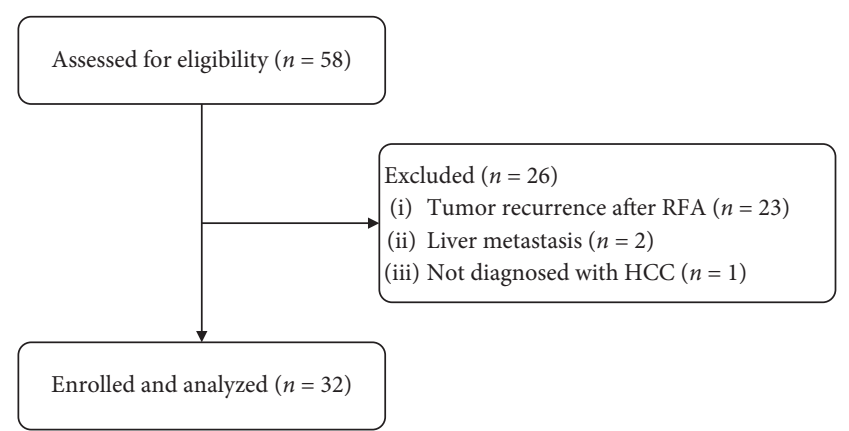

FIgURe 1: Patient selection.

Table 2). However, the dose delivered to the PTV and the tumor characteristics, such as tumor location and diameter, did not contribute to LTC.

The composition of 13 patients with tumor progression is represented in Figure 3, including LTP in 6 patients, IDR in 10 patients, and ER in 3 patients. The median time to progression was not reached either. The one- and two-year PFS rate were $69.9 \%$ (95\% CI, $53.4 \%$ to $86.4 \%$ ) and $52.7 \%$ (95\% CI, 33.1\% to $72.3 \%$ ) (Figure 2(b)), respectively. Multivariate analysis identified higher $\mathrm{CP}$ score as the only independent risk factor for PFS ( $\mathrm{HR}=5.17, P=0.012$, Table 2). Details regarding the lesions showing tumor progression and further treatment modalities are listed in Table 3.

Seven patients died due to tumor progression in the follow-up period. The survival time after SBRT in these seven patients ranged from 4.0 to 29.6 months. The mean survival time of the enrolled patients was 82.9 months (95\% CI 78.4-87.4 months), while the median survival time was not reached. The cumulative 1- and 3-year OS were 85.6\% (95\% CI, $72.5 \%$ to $98.7 \%)$ and $67.1 \%$ (95\% CI, $45.7 \%$ to $88.5 \%)$ (Figure 2(c)). We included LTC as a dependent parameter and found no association between LC and better OS $(\mathrm{HR}=0.85, P=0.88$, Table 2). Age, BMI, liver function, number of prior liver-directed treatments, tumor characteristics, and radiation dosage were not associated with OS.

The results of the univariate and multivariate analyses are shown in Table 2.

3.3. Tolerance and Toxicity. All patients completed their SBRT schedule, and no dose reduction occurred. According to CTCAE 5.0, almost all patients (28 in 32) experienced various sorts and degrees of side effects; among them, only two patients suffered grade 3 adverse events and no patients experienced grade 4 or 5 events. Adverse events are shown in Table 4 . The most common adverse event was decreased platelet count (40.6\%), followed by increased bilirubin (37.5\%) and increased aspartate aminotransferase (34.4\%). No instances of RILD were observed. Only one patient suffered from liver function deterioration, which appeared as a progression of the CP score from 5 to 6 within one month after SBRT. We divided patients into two groups according to the median dose added to the PTV, and Fisher's exact test revealed that there was no correlation between the radiation dosage or liver function and the incidence of
TABLE 1: Baseline characteristics.

\begin{tabular}{|c|c|c|}
\hline Characteristic & No. & $\%$ \\
\hline Total & \multicolumn{2}{|c|}{32} \\
\hline \multicolumn{3}{|l|}{ Sex } \\
\hline Male & 31 & 96.9 \\
\hline Female & 1 & 3.1 \\
\hline \multicolumn{3}{|l|}{ Age (year) } \\
\hline Median (range) & \multicolumn{2}{|c|}{$59.5(29-80)$} \\
\hline \multicolumn{3}{|l|}{ Liver disease } \\
\hline HBV & 22 & 68.8 \\
\hline $\mathrm{HCV}$ & 2 & 6.2 \\
\hline No virus infection & 8 & 25.0 \\
\hline \multicolumn{3}{|l|}{ Anti-HBV therapy } \\
\hline Yes & 20 & 90.9 \\
\hline No & 2 & 9.1 \\
\hline \multicolumn{3}{|l|}{ Cirrhosis } \\
\hline Yes & 15 & 46.9 \\
\hline No & 17 & 53.1 \\
\hline \multicolumn{3}{|l|}{ Child-Pugh score } \\
\hline 5 & 26 & 81.3 \\
\hline 6 & 6 & 18.7 \\
\hline \multicolumn{3}{|l|}{ Prior treatments } \\
\hline Median (range) & \multicolumn{2}{|c|}{$3(1-14)$} \\
\hline RFA only & 11 & 34.4 \\
\hline RFA and surgery & 1 & 3.1 \\
\hline RFA and TACE & 15 & 47.0 \\
\hline RFA, surgery, and TACE & 5 & 15.5 \\
\hline \multicolumn{3}{|l|}{$\operatorname{AFP}(\mathrm{ng} / \mathrm{ml})$} \\
\hline Median (range) & \multicolumn{2}{|c|}{$49.9(1.1-6245.0)$} \\
\hline$\geq 200$ & 12 & 37.5 \\
\hline$<200$ & 20 & 62.5 \\
\hline \multicolumn{3}{|l|}{ Tumor diameter $(\mathrm{mm})$} \\
\hline Median (range) & \multicolumn{2}{|c|}{$28(14-69)$} \\
\hline \multicolumn{3}{|l|}{ Tumor location ${ }^{\dagger}$} \\
\hline Perivessels & \multicolumn{2}{|c|}{23} \\
\hline Subcapsular & \multicolumn{2}{|c|}{8} \\
\hline \multicolumn{3}{|l|}{ Vessel invasion } \\
\hline Yes & 3 & 9.3 \\
\hline No & 29 & 90.7 \\
\hline \multicolumn{3}{|l|}{ Irradiation dose (Gy) } \\
\hline Median (range) & & \\
\hline
\end{tabular}

adverse events ( $P=0.63$ or 0.56 , respectively). Patients who received a higher radiation dosage or had worse liver function did not have a higher risk of enduring adverse events. Furthermore, whether toxicity occurred was not associated with better LTC, PFS, or OS.

\section{Discussion}

Thermal ablation, especially RFA, is recommended in HCC guidelines as one of the curative options for BCLC 0 or A-stage tumors, and the reported $\mathrm{iCR}$ rate of RFA ranges from $83.1 \%$ to $94.8 \%$ [3, 6-8]. Margarita Sala et al. revealed that achievement of an iCR is a major predictor of patient survival after RFA because it is associated with a significant outcome improvement [4]. Tumor size and stage are the 


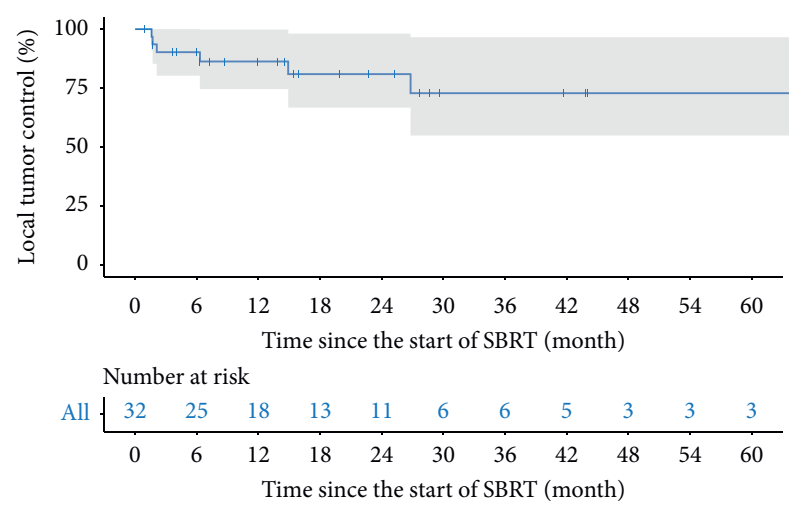

(a)

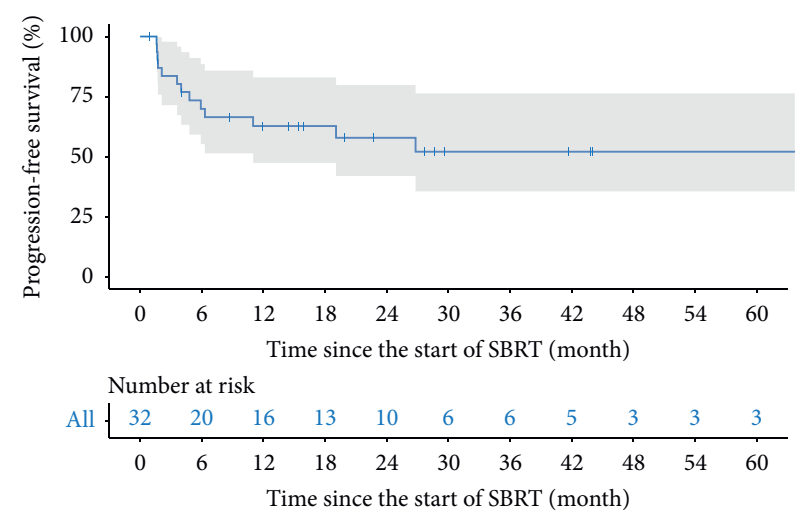

(b)

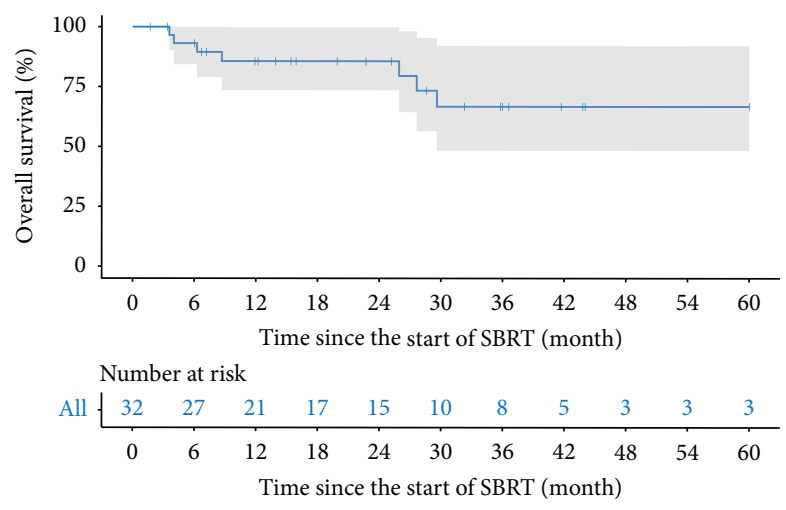

(c)

FIgURE 2: (a) Local tumor control of SBRT. (b) Progression-free survival of SBRT. (c) Overall survival of SBRT.

most important predictors of an $\mathrm{iCR}$, as the $\mathrm{iCR}$ rate reaches approximately $96 \%$ in tumors smaller than $2 \mathrm{~cm}$ and decreases to almost $50 \%$ in case of multinodular or large HCC lesions [4, 22, 23]. In addition, when blood flow is present in the vicinity of the ablation zone, the temperature declines significantly; thus, an iCR is less likely to be achieved. [5]. In most cases, patients receive repeated RFA after the failure of previous RFA procedure $[8,24]$, under which circumstance the limitations mentioned above are not truly resolved. In this observational study, we demonstrate that the strategy of giving individualized SBRT in 6 fractions to patients with a confirmed iIR to previous RFA provided promising tumor control and OS with tolerable adverse events. Six of the 32 patients suffered local progression, and the 1- and 2-year LTC rates were $86.6 \%$ and $74.7 \%$, respectively. Although the LTC was encouraging, SBRT showed less efficiency in overall tumor control; IDR and ER occurred in more than one-third of the enrolled patients, which might have contributed to the unsatisfactory OS. In contrast, studies in which patients enjoyed 2-year post-SBRT LTC and OS rate of 74\% to $95 \%$ and $30 \%$ to $46 \%$, respectively, enrolled large numbers of treat-naïve patients, and patients with residual tumor after RFA composed a small part of their samples $[11,14,17,20]$. Taking the relatively large heterogeneity of those study populations and the underlying distinct response to SBRT into account, we deemed that our study is more representative of patients with an iIR after RFA as well as the ability of
SBRT to treat such residual lesions than the investigations mentioned above.

It has been reported that there exists a radiation doseresponse relationship in HCC: a higher, more intense dose of SBRT likely contributes to a higher rate of LTC $[20,25]$. However, a higher dose delivered to the PTV was not associated with better LTC in the univariate or multivariate analyses in our study. These findings seem contradictory because if a dose-response relationship did exist, then a higher radiation dosage should result in better LTC. A rational explanation could be that, in this investigation, we enrolled a heterogeneous group of patients in terms of the number of prior liver-directed treatments (median, 3; range, from 1 to 14), which indicated that the biological and behavioral characteristics of each tumor nodule differed greatly. Hence, sensitivity to radiation was apparently unique in every malignant lesion, and the benefit of a higher dose in terms of tumor control was concealed. In comparison to previous study, Tse et al. planned an irradiation of $24 \mathrm{~Gy}$ to $54 \mathrm{~Gy}$ (median $36 \mathrm{~Gy}$ ) in six segmentations, the 1year control rate was $65 \%$, along with five patients $(12 \%)$ having grade 3 liver enzyme increases [13]. A trial conducted by Cárdenes et al. reported that when starting at $36 \mathrm{~Gy}$ in 3 fractions, the 1-year control rate reached 100\%, with two patients suffering grade 4 toxicity and three suffering classic RILD [26]. Kang et al. demonstrated that as the radiation dose was increased to $60 \mathrm{~Gy}$ in 3 fractions, the 2-year local 


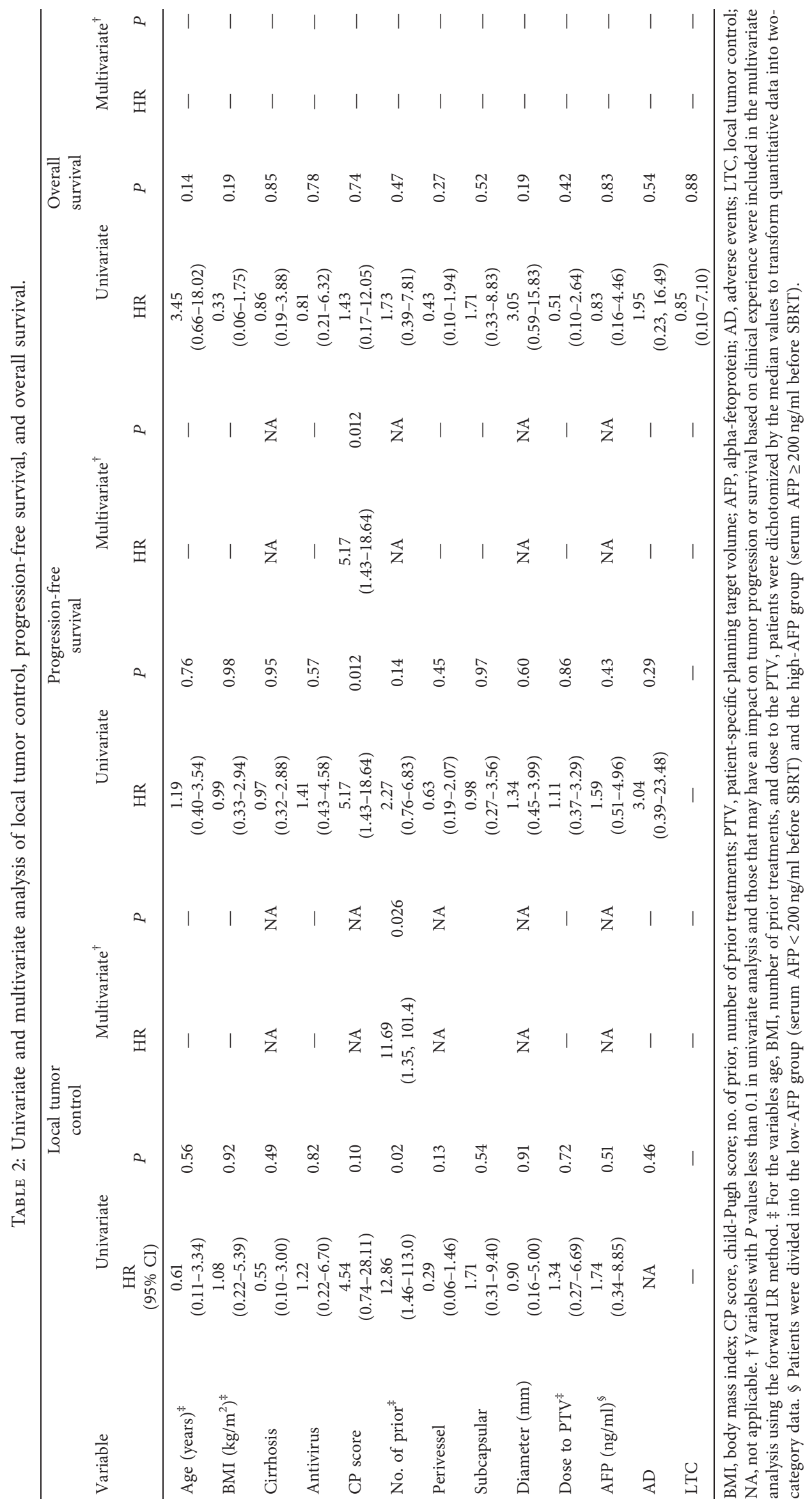




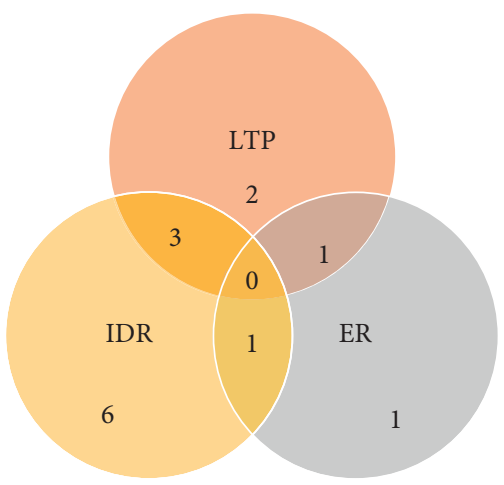

FIgURE 3: Composition of patients with tumor progression. LTP, local tumor progression; IDR, intrahepatic distance recurrence; ER, extrahepatic recurrence.

control rate improved markedly to $94.6 \%$, whereas 2 patients (4.3\%) experienced grade 4 gastric ulcer perforation [27]. In our study, we prescribed a mean dosage of $42 \mathrm{~Gy}$ (30 Gy to $54 \mathrm{~Gy})$ and a 6 -fraction scheme. The 2-year LTC rate was $74.7 \%$, without grade 4 or 5 adverse events or RILD. Combining our research findings with those reported by others, it seems that a lower radiation dose is more tolerable but less efficient for LTC, whereas a higher dose provides better tumor control but with a corresponding higher toxicity. Thus, we recommend $42 \mathrm{~Gy}$ in 6 fractions as a reference schedule for patients with residual diseases after RFA.

Multivariate analysis identified fewer prior treatments to be associated with better LTC. A similar conclusion was drawn in a clinical trial at the University of Michigan Medical Center, where fewer prior liver-directed therapies was related to longer survival [17]. Patients with an iIR after previous RFA received SBRT in our study, but two-thirds of them had received liver-directed therapies before RFA; in other words, two-thirds of the included patients had sustained tumor relapse. HCC in those patients tended to be more aggressive and less responsive to treatments. Accordingly, local tumor recurrence was more likely to occur in those patients. An interesting finding is that LTC had little impact on OS when LTC was included as a variable in the OS analysis ( $\mathrm{HR}=0.85, P=0.88)$. Apart from the diverse stages at which the patients were treated, progression outside the PTV may partly explain this consequence, as even if a single HCC nodule had been permanently eliminated, others would almost inevitably arise $[8,28]$. Thus, combining SBRT with other regional or systemic therapies appears to be rational. For the patient's sake, no matter which local therapy has been adapted, SBRT, RFA, or resection, the regional tumor control rates have been proven similar $[2,7,11-13,17,28-30]$. Therefore, more effective systemic therapies and early recurrence-detection methods are needed.

The CP score was the only significant factor associated with tumor progression in our multivariate analysis. In our study, enrolled patients were all $\mathrm{CP}$ class $\mathrm{A}$, yet those who were rated as CP score 6 were still more likely to suffer tumor progression compared to $\mathrm{CP}$ score 5. A similar finding was reported by Kwon et al., whose study demonstrated the CP score as a significant factor affecting not progression but survival [31]. While health status, tumor burden, and liver function greatly affect the prognosis of HCC patients [2], the $\mathrm{CP}$ score was once considered the strongest prognostic indicator for HCC $[32,33]$. Our result is consistent with the consensus that tumor control is superior in patients with wellpreserved liver function (CP score 5 patients) to those with mild function impairment (CP score 6 patients). Liver function was correlated with tumor control after SBRT in CP class A patients, but what about $\mathrm{CP}$ class $\mathrm{B}$ or $\mathrm{C}$ patients? Most prior studies have recruited $\mathrm{CP}$ class A patients, but Culleton et al. carried out a trial that recruited HCC patients with $\mathrm{CP}$ class B or C liver function. In their trial, the median survival of CP score 7 patients was 9.9 months versus 2.8 months for those $\mathrm{CP}$ score $>7(P=0.011)$, but the difference in time to progression did not reach statistical significance. Moreover, liver toxicity occurred more frequently in patients with $\mathrm{CP}$ class $\mathrm{B}$ or $\mathrm{C}$ liver function. In a phase I study conducted by Cardenes, all CP class A patients received $48 \mathrm{~Gy}$ of SBRT without dose-limiting toxicity, while 3 of $11 \mathrm{CP}$ class $\mathrm{B}$ patients suffered DLT under 42 Gy of radiation [26]. Another retrospective study reported that 2 of $4 \mathrm{CP}$ class $\mathrm{B}$ patients developed RILD following $35 \mathrm{~Gy}$ and $40 \mathrm{~Gy}$ of SBRT [34]. Those studies revealed that $\mathrm{CP}$ class $\mathrm{B}$ or $\mathrm{C}$ patients benefit less but risk more from SBRT; thus, we suggest that SBRT should be considered with caution in patients with high $\mathrm{CP}$ scores, especially CP class B or C patients.

The incidence of grade 3 adverse events was relatively low in our series, and DLT and RILD were not observed. The two patients who had grade 3 anemia and a platelet decrease had baseline hemoglobin and platelet levels close to the lower limits. The adverse effect of SBRT to some degree led to hemoglobin and platelet fall-off; however, the patients' health status contributed to these consequences as well. The two patients' blood system disorders had recovered in the subsequent follow-up. Liver enzyme elevation and bilirubin increase were the most common forms of hepatobiliary toxicity. Liver failure after SBRT was not detected, and all patients were assessed as $\mathrm{CP}$ class A after treatment termination. One patient who received $48 \mathrm{~Gy}$ of irradiation sustained a CP score deterioration from 5 to 6 at the first follow-up. An increase in CP score has often been observed in SBRT studies [11, 13,14, 17, 18, 27, 35], but the exact reasons for this change are not clear. Hepatocyte damage after radiation and tumor progression may contribute to liver function deterioration, which is further reflected by increased total bilirubin, hypoalbuminemia, or CP score progression.

This study has several limitations. As a retrospective observational study, selection bias was inevitable, and little was done to reduce selection bias; thus, our conclusion needs to be further verified by prospective trials. Because of the relatively small sample size, our heterogeneous patient group was not representative enough to achieve a strong statistical power in detecting the effects of the variables. Moreover, some patients were lost to follow-up, and consequently, the long-term influence of SBRT was not fully evaluated, and the survival benefits were not truly presented. 
TABLE 3: Details of patients and lesions with tumor progression.

\begin{tabular}{|c|c|c|c|c|c|c|c|}
\hline ID & Lesion size $(\mathrm{mm})$ & Dose (Gy) & Progression type & PFS time (months) & Further treatment & Follow-up time (month) & Current status \\
\hline 1 & 15 & 36 & LTP & 1.6 & Ablation & 25.9 & DOD \\
\hline 2 & 38 & 39 & IDR & 1.6 & Best Care & 6.0 & Lost \\
\hline 3 & 68 & 39 & IDR & 1.7 & TACE & 6.3 & Lost \\
\hline 4 & 48 & 42 & IDR & 5.9 & TACE & 7.2 & Lost \\
\hline 5 & 29 & 48 & IDR & 11.0 & Ablation & 35.8 & AWD \\
\hline 6 & 25 & 42 & IDR & 19.1 & Ablation & 25.2 & AWD \\
\hline 7 & 20 & 54 & ER & 3.6 & Best Care & 3.6 & DOD \\
\hline 8 & 47 & 48 & LTP + IDR & 2.1 & Best Care & 3.4 & Lost \\
\hline 9 & 69 & 45 & LTP + IDR & 6.3 & TACE & 12.2 & Lost \\
\hline $10^{\dagger}$ & 24 & 39 & $\mathrm{LTP}+\mathrm{IDR}$ & $14.9+4.0^{\dagger}$ & TACE & 36.6 & AWD \\
\hline 11 & 28 & 45 & LTP + IDR & 26.2 & LR & 36.0 & AWD \\
\hline 12 & 19 & 42 & $\mathrm{LTP}+\mathrm{ER}$ & 1.7 & CIK & 1.7 & Lost \\
\hline 13 & 41 & 45 & $\mathrm{IDR}+\mathrm{ER}$ & 4.8 & Best Care & 13.9 & Lost \\
\hline
\end{tabular}

Abbreviations: LTP, local tumor progression; IDR, intrahepatic distance recurrence; ER, extrahepatic recurrence; TACE, transarterial chemoembolization; LR, liver resection; DOD, dead as a result of disease; AWD, alive with disease; Lost, lost to follow-up. $\dagger$ This patient was confirmed to have IDR 4.0 months after SBRT and received TACE as a salvage therapy. LTP was confirmed 14.9 months after SBRT, and TACE was performed on this patient again.

TABle 4: Toxicity of SBRT.

\begin{tabular}{|c|c|c|c|}
\hline \multirow[b]{2}{*}{ Category } & \multicolumn{3}{|c|}{ Toxicity grade } \\
\hline & $\begin{array}{c}\text { Grade } 1 \\
\text { No. }\end{array}$ & $\begin{array}{c}\text { Grade } 2 \\
\text { No. }\end{array}$ & $\begin{array}{c}\text { Grade } 3 \\
\text { No. }\end{array}$ \\
\hline Nausea & 1 & 0 & 0 \\
\hline Abdominal pain & 1 & 0 & 0 \\
\hline Rash & 2 & 0 & 0 \\
\hline Anemia & 7 & 0 & 1 \\
\hline WBC decreased & 5 & 2 & 0 \\
\hline PLT decreased & 8 & 4 & 1 \\
\hline Hypoalbuminemia & 8 & 0 & 0 \\
\hline Bilirubin increased & 10 & 2 & 0 \\
\hline ALT increased & 6 & 0 & 0 \\
\hline AST increased & 10 & 1 & 0 \\
\hline INR increased & 0 & 0 & 0 \\
\hline
\end{tabular}

Abbreviations: WBC, white blood counts; PLT, platelet; ALT, alanine aminotransferase; AST, aspartate aminotransferase; INR, international normalized ratio.

In conclusion, in patients confirmed to have an iIR to prior RFA and compensated liver function, six-fraction SBRT of 42 Gy can provide favorable local tumor control and overall survival along with acceptable toxicity. Our study provides a rationale for clinical trials of SBRT aimed at residual tumor tissue after RFA.

\section{Data Availability}

The data used to support the findings of this study are available from the corresponding author upon request.

\section{Conflicts of Interest}

The authors declare that they have no conflicts of interest.

\section{Authors' Contributions}

Yizhen Fu, Mian Xi, and Yangxun Pan contributed equally to this work.

\section{Acknowledgments}

This work was funded by the National Science and Technology Major Project of China (Nos. 2018ZX10723204 and 2018ZX10302205).

\section{References}

[1] F. Bray, J. Ferlay, I. Soerjomataram, R. L. Siegel, L. A. Torre, and A. Jemal, "Global cancer statistics 2018: GLOBOCAN estimates of incidence and mortality worldwide for 36 cancers in 185 countries," CA: A Cancer Journal for Clinicians, vol. 68, no. 6, pp. 394-424, 2018.

[2] European Association for the Study of the Liver and European Organisation for Research and Treatment of Cancer, "EASL-EORTC clinical practice guidelines: Management of hepatocellular carcinoma," Journal of Hepatology, vol. 69, no. 1, pp. 182-236, 2018.

[3] V. W.-T. Lam, K. K. Ng, K. S.-H. Chok et al., "Incomplete ablation after radiofrequency ablation of hepatocellular carcinoma: analysis of risk factors and prognostic factors," Annals of Surgical Oncology, vol. 15, no. 3, pp. 782-790, 2008.

[4] M. Sala, J. M. Llovet, R. Vilana et al., "Initial response to percutaneous ablation predicts survival in patients with hepatocellular carcinoma," Hepatology, vol. 40, no. 6, pp. 1352-1360, 2004.

[5] J. C. Nault, O. Sutter, P. Nahon, N. Ganne-Carrie, and O. Seror, "Percutaneous treatment of hepatocellular carcinoma: state of the art and innovations," Journal of Hepatology, vol. 68, no. 4, pp. 783-797, 2018.

[6] K. Feng, J. Yan, X. Li et al., "A randomized controlled trial of radiofrequency ablation and surgical resection in the treatment of small hepatocellular carcinoma," Journal of Hepatology, vol. 57, no. 4, pp. 794-802, 2012.

[7] Y.-s. Kim, H. K. Lim, H. Rhim et al., "Ten-year outcomes of percutaneous radiofrequency ablation as first-line therapy of early hepatocellular carcinoma: analysis of prognostic factors," Journal of Hepatology, vol. 58, no. 1, pp. 89-97, 2013.

[8] S. Rossi, V. Ravetta, L. Rosa et al., "Repeated radiofrequency ablation for management of patients with cirrhosis with small hepatocellular carcinomas: a long-term cohort study," Hepatology, vol. 53, no. 1, pp. 136-147, 2011. 
[9] M. Pompili, A. Saviano, N. de Matthaeis et al., "Long-term effectiveness of resection and radiofrequency ablation for single hepatocellular carcinoma $\leq 3 \mathrm{~cm}$. Results of a multicenter Italian survey," Journal of Hepatology, vol. 59, no. 1, pp. 89-97, 2013.

[10] S. S. Lo, A. J. Fakiris, E. L. Chang et al., "Stereotactic body radiation therapy: a novel treatment modality," Nature Reviews Clinical Oncology, vol. 7, no. 1, pp. 44-54, 2010.

[11] A. Bujold, C. A. Massey, J. J. Kim et al., "Sequential phase I and II trials of stereotactic body radiotherapy for locally advanced hepatocellular carcinoma," Journal of Clinical Oncology, vol. 31, no. 13, pp. 1631-1639, 2013.

[12] A. Takeda, N. Sanuki, Y. Tsurugai et al., "Phase 2 study of stereotactic body radiotherapy and optional transarterial chemoembolization for solitary hepatocellular carcinoma not amenable to resection and radiofrequency ablation," Cancer, vol. 122, no. 13, pp. 2041-2049, 2016.

[13] R. V. Tse, M. Hawkins, G. Lockwood et al., "Phase I study of individualized stereotactic body radiotherapy for hepatocellular carcinoma and intrahepatic cholangiocarcinoma," Journal of Clinical Oncology, vol. 26, no. 4, pp. 657-664, 2008.

[14] D. R. Wahl, M. H. Stenmark, Y. Tao et al., "Outcomes after stereotactic body radiotherapy or radiofrequency ablation for hepatocellular carcinoma," Journal of Clinical Oncology, vol. 34, no. 5, pp. 452-459, 2016.

[15] Y. S. Seo, M. S. Kim, H. J. Yoo et al., "Radiofrequency ablation versus stereotactic body radiotherapy for small hepatocellular carcinoma: a Markov model-based analysis," Cancer Medicine, vol. 5, no. 11, pp. 3094-3101, 2016.

[16] J. Chen, K. Peng, D. Hu et al., "Tumor location influences oncologic outcomes of hepatocellular carcinoma patients undergoing radiofrequency ablation," Cancers (Basel), vol. 10, no. 10, 2018.

[17] M. Feng, K. Suresh, M. J. Schipper et al., "Individualized adaptive stereotactic body radiotherapy for liver tumors in patients at high risk for liver damage: a phase 2 clinical trial," JAMA Oncology, vol. 4, no. 1, pp. 40-47, 2018.

[18] S. Culleton, H. Jiang, C. R. Haddad et al., "Outcomes following definitive stereotactic body radiotherapy for patients with child-pugh B or C hepatocellular carcinoma," Radiotherapy and Oncology, vol. 111, no. 3, pp. 412-417, 2014.

[19] R. Lencioni and J. Llovet, "Modified RECIST (mRECIST) assessment for hepatocellular carcinoma," Seminars in Liver Disease, vol. 30, no. 1, pp. 52-60, 2010.

[20] K. E. Rusthoven, B. D. Kavanagh, H. Cardenes et al., "Multiinstitutional phase I/II trial of stereotactic body radiation therapy for liver metastases," Journal of Clinical Oncology, vol. 27, no. 10, pp. 1572-1578, 2009.

[21] J. Kim and Y. Jung, "Radiation-induced liver disease: current understanding and future perspectives," Experimental \& Molecular Medicine, vol. 49, no. 7, p. e359, 2017.

[22] M.-S. Chen, J.-Q. Li, Y. Zheng et al., "A prospective randomized trial comparing percutaneous local ablative therapy and partial hepatectomy for small hepatocellular carcinoma," Annals of Surgery, vol. 243, no. 3, pp. 321-328, 2006.

[23] A. Guglielmi, A. Ruzzenente, S. Pachera et al., "Comparison of seven staging systems in cirrhotic patients with hepatocellular carcinoma in a cohort of patients who underwent radiofrequency ablation with complete response," The American Journal of Gastroenterology, vol. 103, no. 3, pp. 597-604, 2008.

[24] A. Forner, M. Gilabert, J. Bruix, and J.-L. Raoul, "Treatment of intermediate-stage hepatocellular carcinoma," Nature Reviews Clinical Oncology, vol. 11, no. 9, pp. 525-535, 2014.
[25] L. A. Dawson, C. J. McGinn, D. Normolle et al., "Escalated focal liver radiation and concurrent hepatic artery fluorodeoxyuridine for unresectable intrahepatic malignancies," Journal of Clinical Oncology, vol. 18, no. 11, pp. 2210-2218, 2000.

[26] H. R. Cárdenes, T. R. Price, S. M. Perkins et al., "Phase I feasibility trial of stereotactic body radiation therapy for primary hepatocellular carcinoma," Clinical and Translational Oncology, vol. 12, no. 3, pp. 218-225, 2010.

[27] J.-K. Kang, M.-S. Kim, C. K. Cho et al., "Stereotactic body radiation therapy for inoperable hepatocellular carcinoma as a local salvage treatment after incomplete transarterial chemoembolization," Cancer, vol. 118, no. 21, pp. 5424-5431, 2012.

[28] G. N'Kontchou, A. Mahamoudi, M. Aout et al., "Radiofrequency ablation of hepatocellular carcinoma: long-term results and prognostic factors in 235 western patients with cirrhosis," Hepatology, vol. 50, no. 5, pp. 1475-1483, 2009.

[29] R. Lencioni, D. Cioni, L. Crocetti et al., "Early-stage hepatocellular carcinoma in patients with cirrhosis: long-term results of percutaneous image-guided radiofrequency ablation," Radiology, vol. 234, no. 3, pp. 961-967, 2005.

[30] J. Bruix, M. Reig, and M. Sherman, "Evidence-based diagnosis, staging, and treatment of patients with hepatocellular carcinoma," Gastroenterology, vol. 150, no. 4, pp. 835-853, 2016.

[31] J. H. Kwon, S. H. Bae, J. Y. Kim et al., "Long-term effect of stereotactic body radiation therapy for primary hepatocellular carcinoma ineligible for local ablation therapy or surgical resection. Stereotactic radiotherapy for liver cancer," $B M C$ Cancer, vol. 10, p. 475, 2010.

[32] P. Tandon and G. Garcia-Tsao, "Prognostic indicators in hepatocellular carcinoma: a systematic review of 72 studies," Liver International, vol. 29, no. 4, pp. 502-510, 2009.

[33] B. Kok and J. G. Abraldes, "Child-pugh classification: time to abandon?" Seminars in Liver Disease, vol. 39, no. 1, pp. $96-$ 103, 2019.

[34] W.-Y. Huang, Y.-M. Jen, M.-S. Lee et al., "Stereotactic body radiation therapy in recurrent hepatocellular carcinoma," International Journal of Radiation Oncology* Biology ${ }^{*}$ Physics, vol. 84, no. 2, pp. 355-361, 2012.

[35] M. T. Lee, J. J. Kim, R. Dinniwell et al., "Phase I study of individualized stereotactic body radiotherapy of liver metastases," Journal of Clinical Oncology, vol. 27, no. 10, pp. 1585-1591, 2009. 